\title{
A case of male inflammatory breast cancer
}

\author{
Miki Hyakudomi · Toko Inao · Yoshimitsu Minari • \\ Masayuki Itakura $\cdot$ Riruke Maruyama • \\ Yoshitsugu Tajima
}

Received: 10 August 2012 / Accepted: 16 January 2013/Published online: 20 March 2013

(C) The Japan Society of Clinical Oncology 2013

\begin{abstract}
This report describes the case of an 85-year-old male with inflammatory breast cancer. The patient presented with diffuse erythema and induration over the right anterior chest wall. Ultrasonography and mammography demonstrated an ill-defined small mass, $8 \mathrm{~mm}$ in diameter, in the right breast with skin thickening. A core needle biopsy of the breast mass confirmed the presence of invasive ductal carcinoma. A skin biopsy revealed a diffuse tumor cell infiltration with dermal lymphatic emboli. These findings were compatible with the diagnosis of inflammatory breast cancer. The tumor cells were triple negative for estrogen receptor, progesterone receptor, and HER2/neu. His bone scintigraphy showed multiple bone metastases. Systemic chemotherapy using capecitabine was introduced, but it failed to control the disease. TS-1, as second-line systemic chemotherapy, also resulted in treatment failure. Third-line chemotherapy using docetaxel and cyclophosphamide was then administered and was effective. However, he developed pneumonia due to febrile neutropenia after two cycles of treatment and the chemotherapy was discontinued. The patient died of carcinomatous lymphangiosis 2 years and 3 months after the initial onset of the disease. Male inflammatory breast cancer is challenging because of its rarity, biological uncertainness, diagnostic difficulty, and the fact that it is associated with a very poor prognosis. The establishment of a reliable diagnostic and
\end{abstract}

M. Hyakudomi $(\bowtie) \cdot$ T. Inao · Y. Minari · M. Itakura · Y. Tajima

Department of Digestive and General Surgery, Shimane

University Faculty of Medicine, Izumo, Japan

e-mail: mikity@med.shimane-u.ac.jp

R. Maruyama

Department of Pathology, Shimane University Faculty of Medicine, 89-1 Enya-cho, Izumo, Shimane 693-8501, Japan treatment strategy for male inflammatory breast cancer is therefore needed.

Keywords Inflammatory breast cancer - Male .

Docetaxel $\cdot$ Cyclophosphamide

\section{Introduction}

Male breast cancer accounts for less than $1 \%$ of all breast cancer, affecting mainly elderly patients, and is usually discovered after it has progressed significantly [1]. Inflammatory breast cancer is a rare entity, accounting for only $0.5-2 \%$ of all invasive breast cancer, but it has a dismal outcome [2]. Inflammatory breast cancer in men is extremely rare, with only 9 cases having been reported [39]. This report presents a case of male inflammatory breast cancer along with a review of the literature.

\section{Case report}

An 85-year-old male presented with progressive erythema and induration in the right anterior chest wall, which had appeared 6 months earlier. He consulted a dermatologist and was treated with antibiotics before consulting our department. He had a history of hypertension and asthma. There was no family history of breast or ovarian cancer.

A physical examination revealed diffuse erythema and induration in the right anterior chest wall that spread to the right axilla (Fig. 1). His right nipple was retracted, but no masses could be felt. An enlarged lymph node was palpable in his right axilla. Serum level of carcinoembryonic antigen (CEA) was $16.7 \mathrm{ng} / \mathrm{ml}$ (normal range $<5.0 \mathrm{ng} / \mathrm{ml}$ ). All other blood tests were within the normal range. 


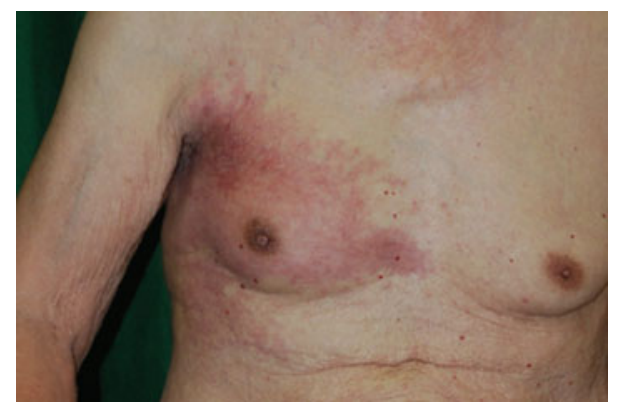

Fig. 1 Erythema and induration in the right anterior chest wall

Mammography and ultrasonography demonstrated an illdefined mass with spiculated margins located in the retroareolar space, $8 \mathrm{~mm}$ in diameter, with cutaneous thickening (Figs. 2, 3). Computed tomography (CT) revealed a centrally located ill-defined mass in his right breast with skin thickening and lymphatic edema from the right anterior chest wall to axilla (Fig. 4). A core needle biopsy confirmed the breast mass to be scirrhous carcinoma (Fig. 5). A skin biopsy noted a diffuse tumor cell infiltration with tumor embolism in the dermal lymphatics, which is characteristic of inflammatory breast cancer (Fig. 6). His bone scan showed multiple sites of increased uptake, being consistent with metastatic disease. No space-occupying lesions were recognized in the lung and liver. The disease was classified as T4dN1M1 stage IV breast cancer. Immunohistochemical stains showed the tumor cells to be negative for both the estrogen receptor (ER) and progesterone receptor (PR).

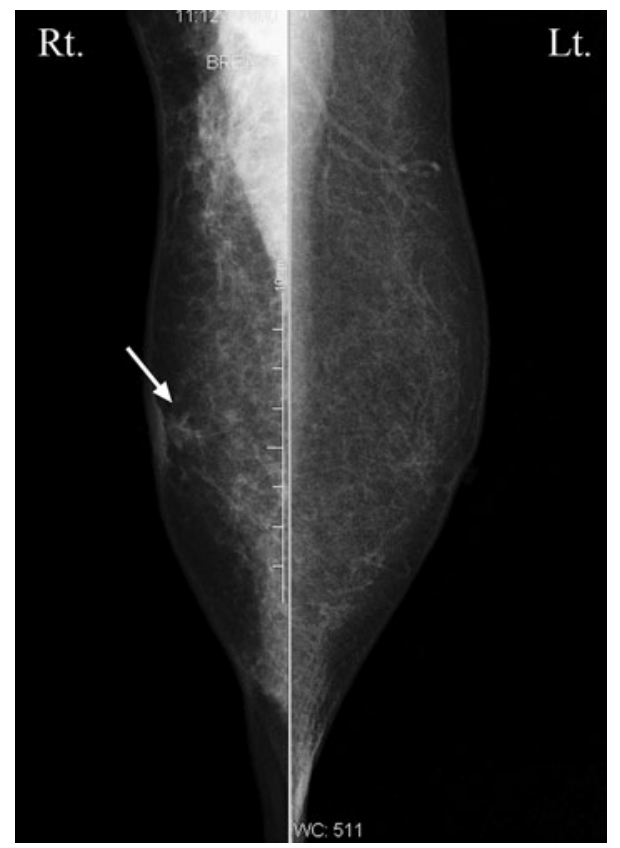

Fig. 2 Mediolateral-oblique (MLO) mammographic views show an ill-defined spiculated mass in the right breast mass (arrow)

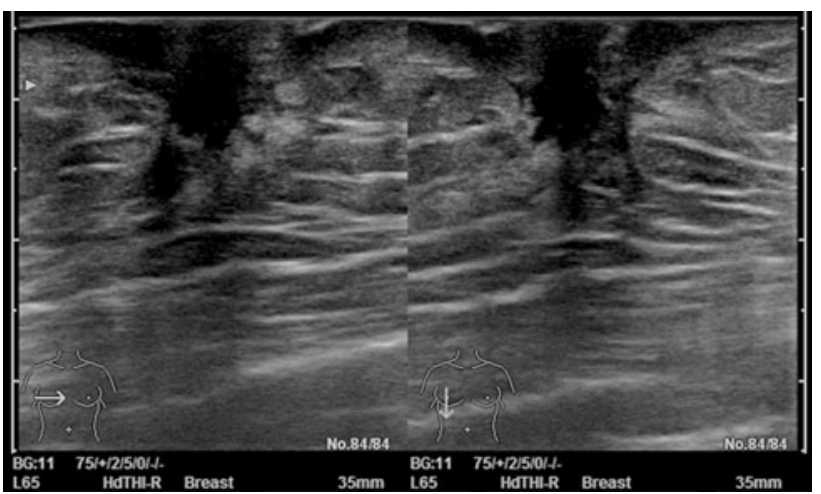

Fig. 3 Ultrasonography shows a low echoic mass in the right retroareolar space

There was no overexpression of human epidermal growth factor receptor 2 (HER2/neu).

Systemic chemotherapy, along with bisphosphonate, was administered using capecitabine $1800 \mathrm{mg}$ /day in two divided doses, with one cycle including 3 weeks of treatment followed by 1 week without treatment. A total of 4 cycles were carried out, but this treatment failed to control the disease. Second-line systemic chemotherapy was introduced using TS-1 $150 \mathrm{mg} /$ day in two divided doses, with one cycle including 4 weeks of treatment followed by 2 weeks without any treatment. A total of 4 cycles were carried out, but this also failed to control the disease and the serum level of CEA increased up to $499.7 \mathrm{ng} / \mathrm{ml}$. Third-line systemic chemotherapy was then administered using TC therapy, i.e., docetaxel (DTX) $60 \mathrm{mg} /$ $\mathrm{m}^{2}+$ cyclophosphamide (CPM) $600 \mathrm{mg} / \mathrm{m}^{2}$. After the TC therapy, his cutaneous symptoms improved and the CEA level was decreased to $28.6 \mathrm{ng} / \mathrm{ml}$. However, he developed pneumonia due to febrile neutropenia after two cycles of TC therapy. Therefore the chemotherapy was discontinued. Although the patient's pneumonia thereafter resolved within 2 months, he was supported with palliation because of his poor performance status. At 3 months after the start of palliative treatment (at 2 years and 3 months after the initial onset of the disease), the patient died of carcinomatous lymphangiosis.

\section{Discussion}

Inflammatory breast cancer is defined as "a clinicopathological entity characterized by diffuse brawny induration and edema of the skin of the breast without a mass", whereas inflammatory breast cancer with a mass is defined as secondary inflammatory breast cancer [10]. In pathological aspects, it is diagnosed by the involvement of the dermal lymphatics with infiltrating cancer cells [2]. The majority of breast cancer patients are women; inflammatory 
Fig. 4 CT demonstrates an illdefined mass (white arrow) in the right breast with skin thickening and lymphatic edema from the right anterior chest wall to axilla (gray arrows)
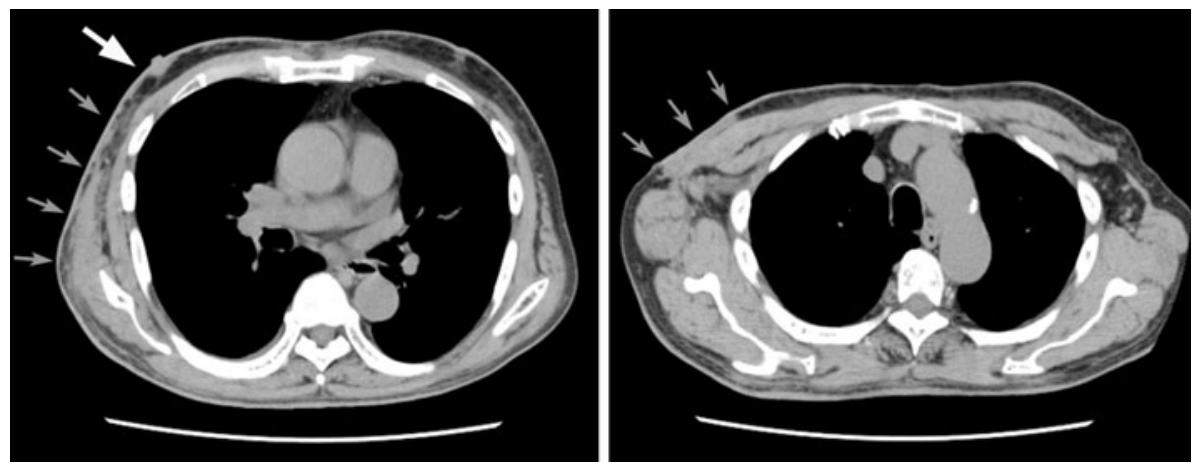

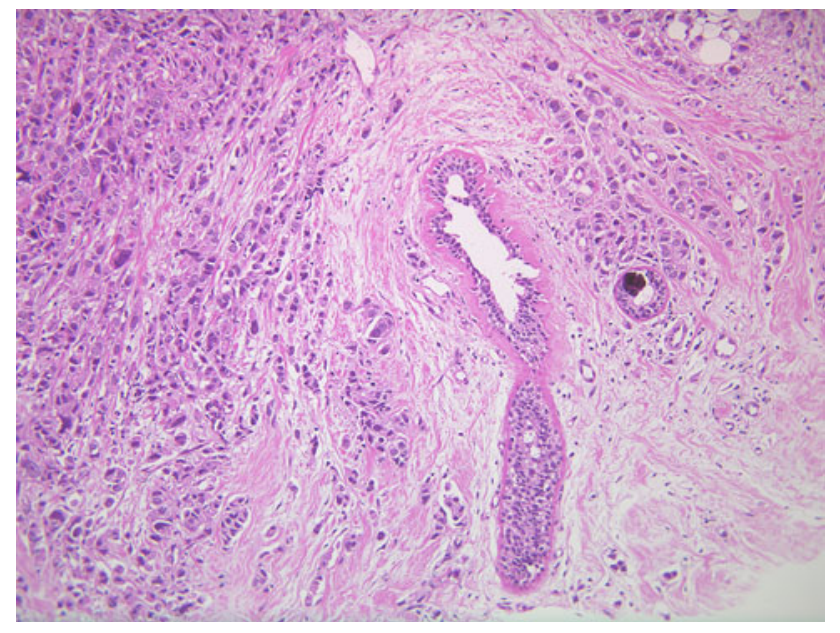

Fig. 5 A biopsy of breast mass reveals scirrhous carcinoma with dermal lymphatic emboli

breast cancer in men is extremely rare, with only 9 cases having so far been reported [3-9].

The clinical details of the 10 male inflammatory breast cancer cases, including the current patient, are summarized in Table 1 . The average age was 67.8 years old. The notable clinical symptoms were a rash and swelling in the chest wall and axillary lymph node swelling. A breast mass was detected by mammography in 3 cases, by ultrasonography in 4 cases, and by CT scans in 3 cases. In our case, a CT scan clearly demonstrated skin thickening and lymphatic edema as well as a small breast mass. The usefulness of contrast-enhanced MRI for making diagnosis of male inflammatory breast cancer was reported in one case [9]. Histological evaluation of the biopsy specimens revealed the infiltrating duct carcinoma in 6 cases and dermal lymphatic emboli in 4. Although an immunohistochemical study was performed on ER and PR in only 3 cases and HER2/neu in 2, tumor cells were negative for both the two hormone receptors and HER2/neu in all cases. A significant portion of the patients with inflammatory breast cancer tend to have ER-negative, HER2-positive, and higher grade tumors compared with other types of breast cancer [11]. On the other hand, the tumor cells observed in male breast cancer are more frequently positive for both ER $(90 \%)$ and PR $(80 \%)$, but they tend to show a lower expression of HER2 (2 \%) [12] than those in female breast cancer [1]. Meanwhile, inflammatory breast cancer in males, including our case, tends to be negative for ER, PR, and HER2,
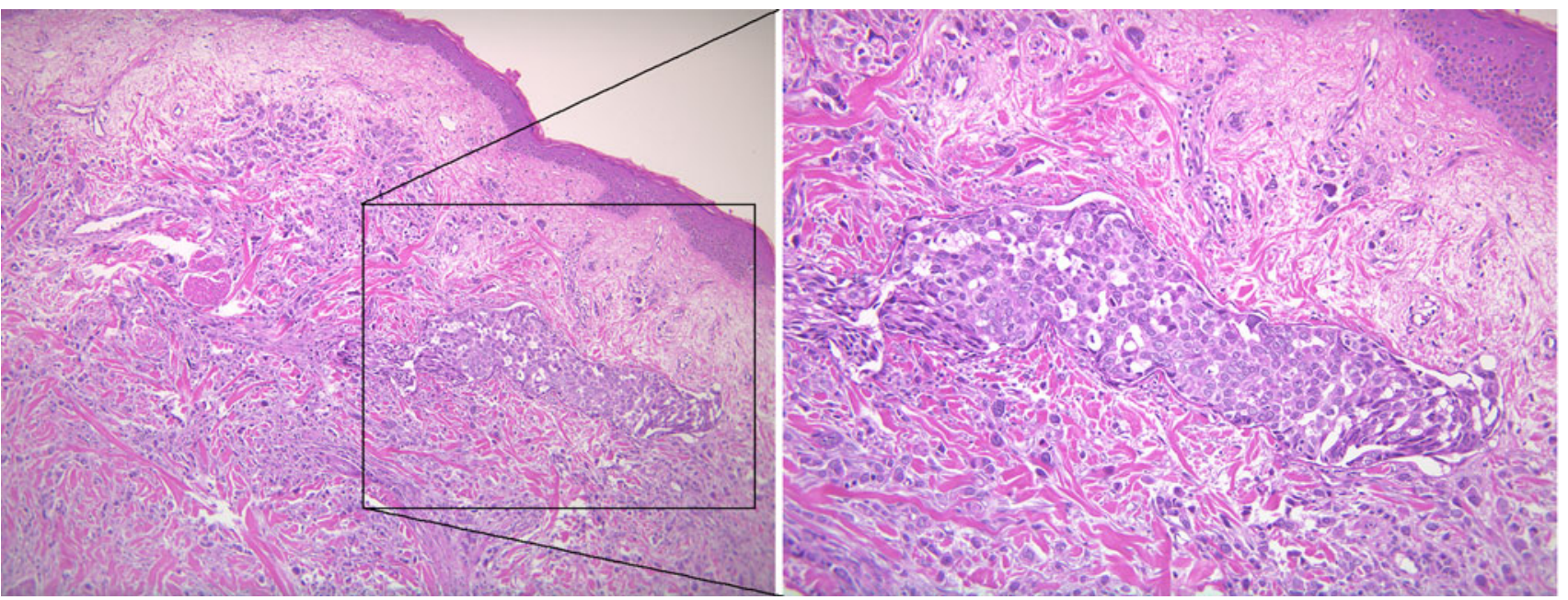

Fig. 6 A skin biopsy demonstrates a diffuse tumor cell infiltration with numerous embolisms in the dermal lymphatics 


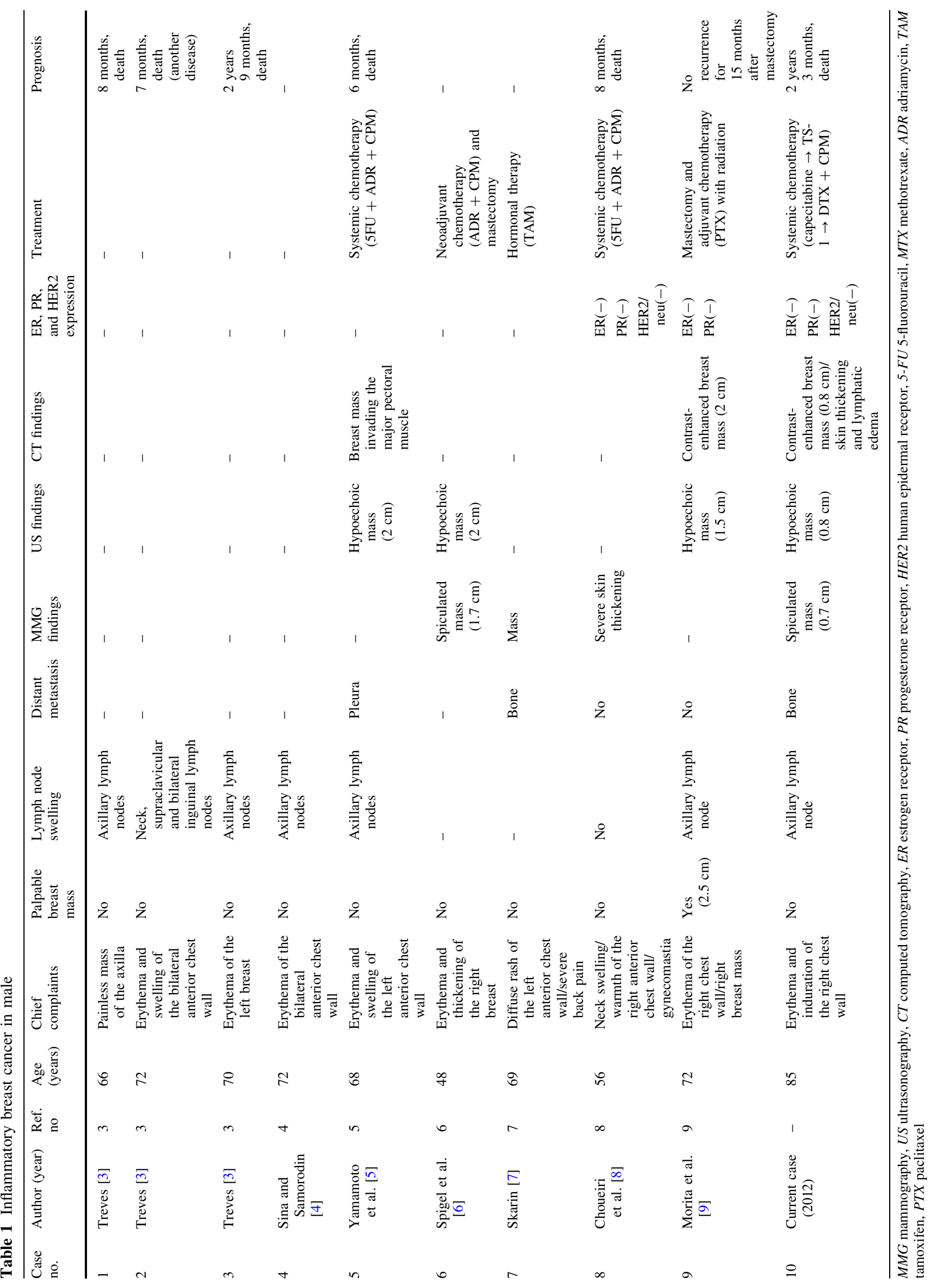


namely the so-called triple negative breast cancer, which may be the reason for their poor prognosis.

Male breast cancer is usually treated with a same strategy to that used for female breast cancer and shows a similar prognosis to female breast cancer [13]. CMF therapy using CPM, MTX, and 5-FU has a response rate of $33 \%$ in male breast cancer and that of CAF therapy using $\mathrm{CPM}, \mathrm{ADR}$, and 5-FU is $63 \%$ [14]. TAM for ER-positive male breast cancer shows a high response rate of $81 \%$ [14]. In patients with inflammatory breast cancer, multimodal treatment including surgery, chemotherapy, and radiotherapy has resulted in improved local disease control and prolonged survival [15]. Chemotherapy using anthracyclines followed by taxanes is thought to be an effective regimen for inflammatory breast cancer [16]. Trastuzumab is also effective for HER2-positive inflammatory breast cancer [16]. The treatment strategies for male inflammatory breast cancer reported in 5 cases (Table 1) are follows: chemotherapy with CAF in 2, hormonal therapy with TAM in one, neoadjuvant chemotherapy with ADR/CPM followed by mastectomy in one, and mastectomy followed by adjuvant chemotherapy with PTX and radiotherapy in one. Two patients treated with CAF showed a slight or no response to the treatment $[5,8]$. The patient who received TAM showed a significant improvement in his skin rash [7]. The patient treated with mastectomy followed by PTX with radiotherapy had survived for 15 months after surgery without recurrence [9]. In our patient, we used oral 5-FU drugs as first-line and second-line chemotherapy because of his advanced age. However, these failed to control the disease. As third-line chemotherapy, TC therapy, i.e., DTX/CPM, with an overall survival benefit in comparison to doxorubicin and cyclophosphamide [17] was thus administered. Although TC therapy has so far only been accepted as an adjuvant regimen, we chose it instead of anthracycline regimens with cardiotoxicity because the patient showed decreased cardiac reserve. TC therapy improved the patient's cutaneous symptoms along with a significant decline in the serum level of CEA. TC therapy was thus thought to be effective for our patient. Unfortunately, he developed pneumonia due to febrile neutropenia after two cycles of TC therapy. As Morita et al. [9] reported the efficacy of PTX for male inflammatory breast cancer, taxanes are expected to become an option for the treatment of male inflammatory breast cancer.

Male inflammatory breast cancer is challenging because of its rarity, biological uncertainness, and diagnostic difficulty. This tumor is mostly discovered at a far-advanced stage in the elderly and its profile of hormone and HER2 receptors is uncertain, thus leading to a poor prognosis. Although TC therapy may be the treatment of choice for this dismal malignant tumor, a clear and reliable treatment strategy for male inflammatory breast cancer still needs to be established.

Conflict of Interest The authors declare that they have no conflict of interest.

\section{References}

1. Fentiman IS, Fourquet A, Hortobagyi GN (2006) Male breast cancer. Lancet 367(9510):595-604

2. Hance KW, Anderson WF, Devesa SS et al (2005) Trends in inflammatory breast carcinoma incidence and survival: the surveillance, epidemiology, and end results program at the National Cancer Institute. J Natl Cancer Inst 97(13):966-975

3. Treves N (1953) Inflammatory carcinoma of the breast in the male patient. Surgery 34(5):810-820

4. Sina B, Samorodin CS (1984) Bilateral inflammatory carcinoma of the male breast. Cutis 33(5):501-502

5. Yamamoto T, Iriyama K, Araki T (1997) Male inflammatory breast cancer. Surg Today 27(7):669-671

6. Spigel JJ, Evans WP, Grant MD et al (2001) Male inflammatory breast cancer. Clin Breast Cancer 2(2):153-155

7. Skarin A (2001) Unusual presentations of malignancy. Case 1. Male inflammatory (?) breast cancer. J Clin Oncol 19(13):3288-3289

8. Choueiri MB, Otrock ZK, Tawil AN et al (2005) Inflammatory breast cancer in a male. N Z Med J 118(1218):1566

9. Morita Y, Syoji T, Goto H, Nakajima et al (2005) A case report of male inflammatory breast cancer (In Japanese). J Jpn Surg Assoc 66(1):36-40

10. The Japanese Breast Cancer Society (2008) General rules for clinical and pathological recording of breast cancer, 16th edn. Kanehara, Tokyo

11. Stephnie S, Sue A, Geraldine W et al (2010) Inflammatory breast cancer-the Royal Marsden Hospital experience. Cancer 1(116):2815-2820

12. Bloom KJ, Govil H, Gattuso P et al (2001) Status of HER-2 in male and female breast carcinoma. Am J Surg 182(4):389-392

13. Borgen PI, Woung GY, Vlamis V et al (1992) Current management of male breast cancer. A review of 104 cases. Ann Surg 215(5):451-457

14. Jaiyesimi IA, Buzdar AU, Sahin AA et al (1992) Carcinoma of the male breast. Ann Intern Med 117(9):771-777

15. Perez CA, Fields JN, Fracasso PM et al (1994) Management of locally advanced carcinoma of the breast. II. Inflammatory carcinoma. Cancer 74(1 Suppl):466-476

16. Dawood S, Ueno NT, Cristofanilli M (2008) The medical treatment of inflammatory breast cancer. Semin Oncol 35(1):64-71

17. Jones S, Holmes FA, O'Shaughnessy J et al (2009) Docetaxel with cyclophosphamide is associated with an overall survival benefit compared with doxorubicin and cyclophosphamide: 7 -year follow up of US oncology research trial 9735. J Clin Oncol 27(8):1177-1183 